\title{
Male attention allocation depends on social context
}

$$
\text { Shelby D. Burridge }{ }^{1} \text {, Ingo Schlupp }{ }^{1} \text {, and Amber M. Makowicz }{ }^{1,2^{*}}
$$

${ }^{1}$ Department of Biology, University of Oklahoma, 730 Van Vleet Oval, Norman, OK 73019, USA

${ }^{2}$ Department of Biological Sciences, Florida State University, 319 Stadium Drive, Tallahassee, FL 32306

${ }^{*}$ Corresponding author's email: amakowicz@bio.fsu.edu

\section{Significance}

This study investigates how attention is allocated in males when presented with social distractions. Assuming that attentional capacity is finite, males may face a tradeoff between different cognitive-demanding stimuli, such as rival males and potential future mates, when mating. Here, we show that male attention allocation in both intra- and intersexual interactions is multifaceted and context dependent. This suggests that individuals within the social environment vary in how meaningful (i.e., able to capture attention) they are to males during mating encounters. Understanding how social partners can cause a shift of attention away from a mating opportunity is essential to understanding the influence of the social context on sexual selection.

\section{Abstract} determine what stimuli are most relevant at a particular moment. In dynamic social environments, multiple individuals may play a pivotal role in any given interaction where a male's attention may be divided between a rival, a current mate, and/or future potential mates. Here, we investigated impacts of the social environment on attention allocation in male sailfin mollies, Poecilia latipinna, which are a part of a sexual-unisexual mating system with the Amazon molly, Poecilia formosa. We asked: 1) Does the species of female influence the amount of attention a male allocates to her? And 2) Is a male's attention towards his mate influenced by different social partners? Males direct more attention toward a stimulus female when she was a conspecific. We also show that males perceive a larger male as a more relevant stimulus to pay attention to than a smaller male, and a conspecific female as a more 
28 relevant stimulus compared to a heterospecific female. Our results show differential allocation of

29 attention is dependent upon multiple components of the social environment in which an individual

30 interacts.

32 Keywords: divided attention, limited attention, sexual-unisexual mating system, social environment,

33 split-attention hypothesis

\section{Introduction}

Attention is one mechanism that organisms can use to screen the flood of information that is transmitted at any moment in time to determine which stimuli are most relevant at a particular moment (1). However, the ability to filter information and provide selective attention is limited. Attentional capacity is constrained by the size of an organism's brain and metabolic costs of neural tissue (2). These limits on attention have been shown to have negative effects on the fitness of an individual

41 when attention has to be divided between several tasks. For example, in blue jays, Cyanocitta cristata, target detection rates for cryptic food items declined when the birds had to divide attention between a peripheral and central feeding location (3). Detection rates continued to decline when birds were presented with an increasing number of distractor items that divided attention further. Other studies have found a balance between dividing attention towards foraging and predator vigilance. In the presence of a predator, feeding rates often decline as attention is given to predators (4), whereas a division of attention towards high foraging rates may lead to an increased likelihood of succumbing to predation (5). Predators themselves face another trade-off and may be less successful feeders due to constraints on attention. When predators confront large groups of prey items, successful predation rates often decline, as the attention of the predator becomes divided among the individual prey items $51(6-8)$.

While there is an increasing body of literature on effects of limited and divided attention on

53 foraging (3), predator avoidance capabilities (4-5), and predation success (6-8), little is known about 54 division of attention in regard to sexual selection. A few studies in humans have shown that sexually 
55 unrestrictive men allocated significantly more attention to attractive women and less to unattractive

56 women (9-10). This allocation of attention towards facial attractiveness seems to be dependent upon sociosexuality, relationship status, and perceived self-market value of the choosers (11). Similarly, in animals that live in social groups, several individuals may play a role in any given interaction. When a male is interacting with a particular female, other individuals such as rival males and potential future mates may be present that draw attention away from the current interaction (12-13). In the present study, we investigated how males will divide their attention between a stimulus female and a variable audience to determine how the context of the social environment affects attention allocation, assuming that attentional capacity is finite.

Measuring attention allocation can be challenging in non-primate models where typical methods such as fMRIs (14), event-related potential (11), and eye tracking glasses (15-16) are less feasible options. Most studies, therefore, use other, indirect measurements, particularly the proportion of time allocated to specific tasks (17-18), latency to start a particular task $(4,5,17)$, distance to prey before a predator is detected (19), or the number of correct responses $(3,20-21)$. The time animals spend allocating between two separate tasks (e.g., the tradeoff between predator vigilance and

70 foraging; 18) can be a reliable measure of how they allocate attention and balance their cognitive load.

71 Studying how social distractions influence attention allocation within the context of sexual selection in non-primates can be even more challenging, as it can use the same units of measure (e.g., proportion of time) as in other components of sexual selection (e.g., mate preference). However, even in humans, the proportion of time was used to measure how much attention individuals allocated to attractive opposite-sex faces (9). Thus, we apply this same methodology to study how attention is allocated between the proportion of time males can spend mating with a readily accessible, fertile female and the time he spends interacting with a rival male or a potential, but inaccessible mate. Specifically, we measure the tradeoff between intrasexual competition and mating, where a sexually motivated male is predicted to spend more time physically interacting with a fertile female over visually interacting with 
81 potential mate, where a sexually motivated male is predicted to spend more time physically interacting

82 with a fertile, accessible female over visually interacting with an inaccessible potential mate. toward rival males (22). Sailfin mollies provide an interesting system to study attention allocation because in addition to their highly dynamic social environment, they are also host to a sexual parasite, the Amazon molly, Poecilia formosa. Amazon mollies are gynogenetic, unisexual hybrids that arose from a natural hybridization event between sailfin mollies and the Atlantic molly, Poecilia mexicana;

thus, they occur syntopic with their host species (23-24). Male sailfin mollies are capable of distinguishing conspecific females from heterospecific Amazon mollies and show preference towards conspecifics (25-27). The use of the sexual-unisexual mating system of sailfin mollies to address questions regarding effects of the social environment on attention allocation provides another

Does the species of female influence the amount of attention a male allocates to her? And 2) Is a male's attention towards his mate influenced by different social partners? Specifically, we asked how a focal male divides attention between a stimulus female (either a conspecific or heterospecific female) with whom he can readily interact with and either a rival male which varied in body size or a potential 101 mate (i.e., a conspecific or heterospecific female). To study how intrasexual competition influenced 102 the attention allocation of male sailfin mollies we used eight social conditions: 1) sailfin molly female 103 with smaller-sized male audience; 2) sailfin molly female with larger-sized male audience; 3) sailfin 104 molly female with equally sized male audience; 4) sailfin molly female with no audience; 5) Amazon 105 molly female with smaller-sized male audience; 6) Amazon molly female with larger-sized male 106 audience; 7) Amazon molly female with equally sized male audience; and 8) Amazon molly female 107 with no audience. To assess how potential future mates influence male attention we simulated six 
108 social conditions in which a focal male observed: 1) a sailfin molly stimulus female with sailfin molly

109 audience female; 2) a sailfin molly stimulus female with Amazon molly audience female; 3) a sailfin

110 molly female with no audience; 4) an Amazon molly stimulus female with sailfin molly audience female;

111 5) an Amazon molly stimulus female with Amazon molly audience female; and 6) an Amazon molly

112 stimulus female with no audience.

113 If attention allocation occurs randomly, we would expect males to split their attention equally

114 between the stimulus female and the audience individual. However, if attention allocation is dependent

115 upon the relative information value of the stimuli, we predicted that males would allocate more attention

116 overall towards the more valuable stimulus female (i.e., the conspecific female), compared to the less

117 valuable stimulus female (i.e., the heterospecific female). In the intrasexual competition social 118 conditions, we predict that the similar-sized males would detract the greatest amount of the focal 119 male's attention away from the stimulus female (28), there will be little detraction from smaller males 120 (29-30), and males that are larger than the focal male will result in focal males spending less attention 121 towards a stimulus female in order to conceal his preference toward that female and reduce the 122 likelihood that the audience male will copy his mate choice (31-32). For the potential future mate social 123 conditions, we predicted that males would allocate equal amounts of attention when both females are 124 conspecifics, more attention towards the conspecific audience female when paired with a 125 heterospecific stimulus female, and more attention towards the conspecific stimulus female when 126 paired with a heterospecific audience female (25-27).

\section{Results}

129 (a) Effects of intrasexual competition on male attention

130 When analyzing the actual time males spent giving attention to the stimulus female, we found 131 that males spent more time with a stimulus female when she was conspecific and when there was no 132 audience present (Tables 1, 2, SI Appendix, Fig. S1, Table S1). This was generally predicted. Males 133 spent the most time interacting with the audience male when paired with a heterospecific compared 134 to a conspecific female. Unsurprisingly, males spent the least amount of time interacting near the 
135 Plexiglas container when there was no audience present (Tables 1, 2, SI Appendix, Fig. S1, Table 136 S1).

137 When we investigated the proportion of time males spent with either the stimulus female or 138 audience male, we found a significant effect on the direction males allocated their attention $139\left(F_{1,17}=73.039, p<0.001, \eta^{2}=0.811\right)$, with males allocating more attention towards the stimulus female 140 (Fig. 1). Neither the species of stimulus female $\left(F_{1,17}=0.900, p=0.356, \eta^{2}=0.050\right)$ nor the audience 141 male type $\left(F_{2,16}=0.117, p=0.891, \eta^{2}=0.014\right)$ caused a significant difference. However, there was a 142 significant interaction between the direction of allocated attention and the stimulus female type $143\left(F_{1,17}=7.634, p=0.013, \eta^{2}=0.310\right)$ and the audience male type $\left(F_{2,16}=4.959, p=0.021, \eta^{2}=0.383\right)$. Males 144 allocated more attention towards the stimulus female when she was a conspecific compared to a 145 heterospecific $(p=0.030)$ and more attention towards the audience when the stimulus female was a 146 heterospecific compared to a conspecific ( $p=0.037$; electronic supplementary material, table S3). 147 Larger male audiences increased the amount of allocated attention towards the audience male 148 compared to the smaller male audience $(p=0.003)$ or an audience male equal in size $(p=0.005)$. Males 149 directed more attention towards the stimulus female when in front of a smaller male audience 150 compared to a larger male $(p=0.020)$. Finally, there was no significant interaction between the type of 151 stimulus female and the type of audience male $\left(F_{2,16}=0.133, p=0.877, \eta^{2}=0.016\right)$ or the direction of 152 attention, type of stimulus female, and the type of audience male $\left(F_{2,16}=1.536, p=0.245, \eta^{2}=0.161\right)$.

(b) Effects of a potential mate on male attention

Again, sailfin males spent most their attention towards conspecific stimulus females and spent

156 the most time with the stimulus female when he had no distractions compared to when there was an 157 audience present (Tables 1, 3; SI Appendix, Fig. S2, Table S3). Male sailfin mollies directed more 158 attention towards the audience when she was a conspecific female, but there was no effect of the 159 stimulus female type on audience-directed attention. However, there was a significant interaction 160 between the stimulus female type and audience female type, with males spending almost twice as 
161 much time with the audience female when that female was a conspecific and the stimulus female was

162 a heterospecific (Tables 1, 3; SI Appendix, Fig. S2, Table S3).

163 When we investigated the proportion of time males spent with either the stimulus female or

164 audience female, we found a significant effect of the direction of allocated attention $\left(F_{1,19}=23.724\right.$,

$\left.165 p<0.001, \eta^{2}=0.555\right)$ with males spending more attention on the stimulus female (Fig. 2). There was

166 also a significant effect of stimulus female type $\left(F_{1,19}=4.922, p=0.039, \eta^{2}=0.206\right)$, where males

167 allocated more attention towards conspecific females than heterospecific females. The type of

168 audience female had no significant effect on attention allocation $\left(F_{1,19}=1.471, p=0.240, \eta^{2}=0.072\right)$.

169 There was a significant interaction between the direction of allocated attention and the type of stimulus

170 female $\left(F_{1,17}=4.596, p=0.045, \eta^{2}=0.194\right)$, with males spending more time with the stimulus female if

171 she was a conspecific $(p=0.019)$. The direction of allocated attention also had a significant interaction

172 with the type of audience female $\left(F_{1,19}=4.574, p=0.046, \eta^{2}=0.194\right)$, with males allocating more

173 attention towards the audience female if she was a conspecific female $(p=0.021 ; S /$ Appendix, Table

174 S4). While there was no significant interaction between the type of stimulus female and the type of

175 audience female $\left(F_{1,19}=0.609, p=0.445, \eta^{2}=0.031\right)$, there was an overall significant interaction between

176 the direction of allocated attention, the type of stimulus female, and the type of audience female

$177 \quad\left(F_{1,19}=4.934, p=0.039, \eta^{2}=0.206\right)$.

178

179

180

181

182

183

\section{Discussion}

The general presence of an additional social partner, regardless of the sex, size, or species, led to a significant decrease in the attention (measured as time) males allocated to the stimulus female. The general effect of divided attention in both the intrasexual competition and potential mate experiments are unsurprising. When the number of items an individual has to focus on increases, the amount of time and effort an individual can allocate to each is significantly reduced due to neurological constraints $(20,33-34)$. This is especially true here: the amount of time a male allocated towards the stimulus female in the control treatments was significantly higher when compared to any of the audience treatments (Fig. 1, 2). Similar effects of attention divided among multiple individuals have 
188 been found in other species. For instance, in Allenby's gerbils, Gerbillus andersoni allenbyi, an

189 individual's attention may be divided between the presence of a predator and the density load of

190 ectoparasites resulting in a dramatic decrease in time allocated to foraging (18). In the cichlid

191 Lamprologus ocellatus, males were shown to divide their attention between predator vigilance and

192 either a conspecific or heterospecific intruding male, and when males were interacting with other

193 conspecific males there was a significant reduction in the distance for males to detect predators (19).

194 Similarly, we show that in the context of sexual selection (i.e., male-male competition and female mate-

195 choice copying), there is also a division of attention between the individuals within the subject male's

196 immediate social environment regardless of the specific type of individuals. Furthermore, the species

197 of stimulus female is the strongest predictor on how males allocate their attention. In all of the

198 combinations of stimulus female type and audience type, sailfin molly males preferred to interact with

199 conspecific rather than heterospecific females. This result is not unexpected as it has been

200 documented in previous studies that sailfin males prefer conspecific females over heterospecifics (25-

$20126,35)$. This may also imply that it is more beneficial to the fitness of an individual male to allocate his

202 attention toward a possible mate than to compete with a potential rival or interact with other potential

203 mates.

204 Contrary to our predictions, males spent significantly more time associating with a larger male

205 than they did with similar-sized males regardless of female stimulus species. Indeed, focal males

206 tended to interact more with the audience when the male was larger than himself compared to either

207 of the other two male size classes. Again, this result is not completely unexpected, given that females

208 within the system prefer larger males over smaller males, and provide more evidence that female

209 choice rather than male competition leads to mating opportunities (28-30). Had the audience and the

210 stimulus male been able to physically interact rather than only being able to interact visually and

211 chemically, these results may have been different. It remains unknown for this study if this switch in

212 attention from the stimulus female to the larger audience male is a way for males to conceal their mate

213 preference or something else altogether (36). Conversely, males appeared to allocate more time

214 toward the stimulus female when the audience male was smaller than the focal male. This result may 
215 be due in part to a lower sperm competition risk posed by a smaller male or a male close to the size

216 of the focal male and an increase in male-male competition pressure by a larger male (29-30).

217 However, the result may also imply that males perceive a larger male as a more relevant stimulus than

218 a smaller male, leading to differential division of attention. Finally, association time with the male

219 audience appeared to be influenced by stimulus female type. Males spent more time with the audience

220 male when the stimulus female was a heterospecific than they did when the stimulus female was a

221 conspecific. Since Amazon mollies do not provide a direct benefit to male fitness $(23,37)$, male

222 attention was divided more than when a conspecific female is presented as a stimulus. Nonetheless,

223 these results are evidence that male sailfin mollies are somehow aware of their own body size and

224 can compare his relative size to other males. Furthermore, from this self-reference comparison, males

225 can then alter their attention according to their perceived competition.

226 There was no overall effect of female audience type on male attention allocation towards the

227 stimulus female beyond the initial decline of adding a second individual. Since the stimulus females

228 are more readily accessible than the audience females (who were enclosed in a chamber), males were

229 able to direct attention towards the stimulus female regardless of audience species in order to gain

230 immediate copulations and increase the possibility that an audience female will copy the mate choice

231 of the stimulus female in future reproductive encounters. While there was no effect of the type of

232 audience female on how much attention males allocated to the stimulus female, there was a significant

233 effect of audience on the amount of attention allocated towards the audience female. Males spent

234 significantly more time interacting with the more valuable conspecific audience compared to a

235 heterospecific audience. This effect was greatest when the stimulus female was a heterospecific

236 Amazon molly.

237 The results provided by this study show that division of attention is not determined by one

238 factor alone, but rather the components of the social environment in which the organism is interacting.

239 Social context mediates allocation of attention so that an individual gives the greatest amount of

240 attention to the most relevant stimuli at a given moment, whether that is toward a rival male, an

241 accessible female, or a possible future mate. Furthermore, the social partners seem to vary in how 
242 meaningful they are to males during mating encounters. Stimuli that have meaning to the individual

243 are better able to capture attention, whereas stimuli that lack meaning fail to (38). Thus, in order to

244 capture attention, upstream cognitive processing must occur to determine the relevance of said

245 stimulus prior to directing attention towards it (39). Our data suggests that the information regarding

246 the body size of a male competitor and the species of a potential mate is being processed by the brain

247 and found to have meaning in the context of current mating encounters (although we did not include

248 a social stimulus that had no meaning to the male). These different social partners also seem to vary

249 in the degree of meaningfulness, as indicated by the differences in attention spent towards each.

250 Understanding what qualities of rival males or potential mates have meaning to males enough to cause

251 a shift of attention away from a mating opportunity is essential to understanding the influence of the

252 social context on sexual selection. In addition, incorporating more social experience and/or social

253 structure into how attention is allocated would provide more information on how the social environment

254 influences social attention and cognition (40). Our study shows that division of attention is multifaceted

255 and context dependent, however, much more work should be done to elucidate a broader range of

256 social implications and the limits of being able to divide attention during sexual and competitive

257 interactions.

258 Methods

259 (a) Study subjects

$260 \quad$ Subjects were collected from a drainage basin in Weslaco, Texas $\left(26^{\circ} 7^{\prime} 13.13^{\prime \prime} \mathrm{N}\right.$

$26197^{\circ} 57^{\prime} 41.08^{\prime \prime W}$ ) and brought back to the University of Oklahoma, Norman, OK, USA. Collection trips

262 were conducted in May 2015, July 2015, October 2015 and May 2016. Fish were housed in small

263 mixed-sex/mixed-species groups in 37.85-L tanks under 12L/12D light conditions. Fish were allowed

264 to acclimate to laboratory housing for a minimum of 30 days before testing began. After the 30 -day

265 acclimation period, measurements of standard length $(\mathrm{mm})$ were taken for all males who were

266 subsequently placed in individual 3.79-L isolation tanks and isolated from females for more than 2

267 weeks to increase their sexual drive (41). Female fish were then separated by species into 37.85-L

268 tanks to await testing. 
(b) Experimental setup

All trials were conducted in an experimental tank (37.85-L), which had white Plexiglas sheets lining three sides to prevent external distractions. The fourth, long side of the tank remained visible in order to film the behavioral interactions (Nikon D5200 24.1 MP CMOS Digital SLR camera at Full HD

$274(1080 p))$. A clear, perforated Plexiglas container was placed in the rear center of the tank to house the 275 audience individual (a male or female individual, see below), while two additional perforated Plexiglas 276 containers were placed in the center of the tank to individually house the focal male and stimulus female. After a 10-minute acclimation period, the subject male and stimulus female were released and permitted to interact for 10 minutes. After this period, both the subject male and stimulus female were returned to the Plexiglas containers to await the next trial (see below), or if all trials were complete, both individuals were returned to their former holding tanks. All videos were then analyzed by recording 281 the amount of time (s) a male spent interacting with either the stimulus female or the audience 282 individual.

For this experiment, we assessed how intrasexual competition and potential future mates influenced focal male attention by comparing association times (i.e., the time males spent with the stimulus female compared to the time spent with the audience individual). To assess how intrasexual competition influenced male attention towards the stimulus female, we used audience males who varied in body size relative to the focal male (i.e., a male that was within $2 \mathrm{~mm}$ of standard length compared to the subject male, a male that was $4 \mathrm{~mm}$ smaller than the subject male, and a male that 289 was $4 \mathrm{~mm}$ larger than the subject male). To assess how potential future mates influenced focal male 290 attention, we used an audience female that was either a conspecific (female sailfin molly) or a 291 heterospecific (Amazon molly). Each trial consisted of a subject male paired with either a conspecific 292 or heterospecific stimulus female with an audience individual present. For the intrasexual competition 293 trials, subject males participated in a total of eight social conditions over a two-day period. For the 294 potential mate trials, subject males participated in six social conditions over a two-day period. The two days were split according to stimulus female species, which was chosen at random. On that day, a 
296 male would undergo the control for that female species and the three male size trials, which were

297 randomized within that day. All females were size matched to a standard length within $\pm 3 \mathrm{~mm}$. We

298 used the same males for both experiments, however, each male was given a one-week rest between

299 experiments.

$301 \quad$ (c) Statistical analyses

302 To assess the time that males allocate to the stimulus female and the audience individual, we 303 evaluated two different data sets for each experiment. In the first data sets, we evaluated the actual 304 time that males allocated to either the stimulus female or the audience individual from the raw data in 305 order to compare the time spent with either individual to the control treatment. Our second data set, 306 we wanted to directly compare the total amount of attention a male allocated towards the stimulus 307 female compared to the audience individual from the total available time. Specifically, we investigated 308 the proportion of time by dividing the raw time spent with each by the total available time (600 s). 309 These values were then arcsin(square root) transformed to fit normality and used in subsequent 310 repeated measures general linear models (GLM).

311 For the intrasexual competition trials a total of 18 males were tested in each of the 8 312 treatments, and for the potential mate trials, a total of 20 males were tested in each of the 6 treatments.

313 To assess effects of audience on attention allocation, we ran three repeated measures general linear 314 models (GLM) for the intrasexual competition and potential mate trials. In our first mixed model, male 315 identity was used as a fixed factor to account for variation between individual males while stimulus 316 female type (conspecific or heterospecific) and audience type (intrasexual competition trials: control, 317 smaller than, equal to or larger than focal; potential mate trials: conspecific or heterospecific) were 318 used as fixed factors. The response variable was association time (s) with the stimulus female. In the 319 second model, the random and fixed factors remained the same, while the response variable was 320 association time (s) with the audience individual. The third model tested the proportion of time males 321 spent with either the stimulus female compared to the audience out of the total time available. We used a repeated measures GLM with male identity as the repeated measure, and our fixed factors 
323 were attention direction (to stimulus female or audience individual) stimulus female type (conspecific

324 or heterospecific), and audience type. In this model, our response variable was the proportion of time

325 males allocated towards the stimulus female. All models included Bonferroni-corrected post hoc tests

326 to further compare the audience treatments and interactions. All data analysis was conducted using

327 SPSS (IBM v. 24).

328

329

330

331

332

333

334

335

336

337

338

339

340

341

342

343

344

345

346

347

348

349

350

351

352

353

354

Acknowledgements. We would like to thank Rachel Steele, Elizabeth Hardy, Tana Moore, and Parker Fleming with their assistance on the collection trips and fish maintenance and Texas Parks and Wildlife (permit \# SPR-0305-045) for collection permits. This research is in partial fulfillment of MS thesis requirements for SDB.

\section{References}

1. S.J. Shettleworth, Cognition, evolution, and behavior. (Oxford Univ. Press., Oxford, 2010)

2. R. Dukas, Causes and consequences of limited attention. Brain Behav. Evol. 63, 197-210 (2004).

3. R. Dukas, A.C. Kamil, The cost of limited attention in blue jays. Behav. Ecol. 11, 502-506 (2000).

4. M. Milinski, R. Heller, Influence of a predator on the optimal foraging behaviour of sticklebacks (Gasterosteus aculeatus L.). Nature 275, 642-644 (1978).

5. J.-G.J. Godin, S.A. Smith, A fitness cost of foraging in the guppy. Nature 333, 69-71 (1988).

6. D.C. Krakauer, Groups confuse predators by exploiting perceptual bottlenecks: a connectionist model of the confusion effect. Behav. Ecol. Sociobiol. 36, 421-429 (1995).

7. L. Landeau, J. Terborgh, Oddity and the 'confusion effect' in predation. Anim. Behav. 34, 13721380 (1986).

8. R.C. Miller, The significance of the gregarious habit. Ecology 3,122-126 (1922).

9. J.K. Manor, D.T. Kendrick, D.V. Becker, A.W. Delton, B. Hofer, C. Wilbur, S. Neuberg, Sexually selective cognition: Beauty captures the mind of the beholder. J. Pers. Soc. Psychol. 85, 11071120 (2003).

10. L.A. Duncan, J.H. Park, J. Faulkner, M. Schaller, S.L. Neuberg, D.T. Kenrick, Adaptive allocation of attention: Effects of sex and sociosexuality on visual attention to attractive opposite-sex faces. Evol. Hum. Behav. 28, 359-364 (2007).

11. L.K. Morgan, M.A. Kisley, The effects of facial attractiveness and perceiver's mate value on adaptive allocation of central processing resources. Evol. Hum. Behav. 35, 96-102 (2014).

12. T.J. Valone, From eavesdropping on performance to copying the behavior of others: a review of public information use. Behav. Ecol. Sociobiol. 62, 1-14 (2007). 
13. C.A.F. Wascher, J.W. Valdez, C. Núñez Cebrián, V. Baglione, D. Canestrari, Social factors modulating attention patterns in carrion crows. Behaviour. 151, 555-572 (2014).

14. J.C. Culham, P. Cavanagh, N.G. Kanwisher, Attention response functions: Characterizing brain areas using fMRI activation during parametric variations of attentional load. Neuron 32, 737-745 (2001).

15. K. Shavit-Cohen, E.Z. Golumbic, The dynamics of attention shifts amony concurrent speech in a naturalistic multi-speaker virtual environment. Fron. Hum. Neurosci. 13, 386 (2019).

16. N. Gomes, G.R. Semin, Mapping human vigilance: The influence of conspecifics. Evol. Hum. Behav. 41, 69-75 (2020).

17. S.W. Griffiths, S. Brockmark, J. Höjesjö, J.I. Johnsson, Coping with divided attention: The advantage of familiarity. Proc. R. Soc. Lond. B 271, 695-699 (2004).

18. A. Raveh, B.P. Kotler, Z. Abramsky, B.R. Krasnov, Driven to distraction: detecting the hidden costs of flea parasitism through foraging behaviour in gerbils. Ecol. Let. 14, 47-51 (2011).

19. K. Ota, Fight, fatigue and flight: Narrowing of attention to a threat compensates for decreased antipredator vigilance. J. Exp. Biol. 221, jeb168047 (2018).

20. R. Dukas, A.C. Kamil, Limited attention: the constraint underlying search image. Behav. Ecol. 12, 192-199 (2001).

21. S. Gomes-Ng, D. Elliffe, S. Cowie, Relative reinforcer rates determine pigeon's attention allocation when separately trained stimuli are presented together. Learn. Behav. 47, 245-257 (2019).

22. A.D. Woodhead, N. Armstrong, Aspects of the mating behaviour of male mollies (Poecilia spp.). J. Fish Biol. 27, 593-601 (1985).

23. C.L. Hubbs, L.C. Hubbs, Apparent parthenogenesis in nature, in a form of fish of hybrid origin. Science 76, 628-630 (1932).

24. M. Stöck, K.P. Lampert, D. Möller, I. Schlupp, M. Schartl, Monophyletic origin of multiple clonal lineages in an asexual fish (Poecilia formosa). Mol. Ecol. 19, 5204-5215 (2010).

25. C.R. Gabor, M.J. Ryan, Geographical variation in reproductive character displacement in mate choice by male sailfin mollies. Proc. R. Soc. B 268, 1063-1070 (2001).

26. M.J. Ryan, L.A. Dries, P. Batra, D.M. Hillis, Male mate preferences in a gynogenetic species complex of Amazon mollies. Anim. Behav. 52, 1225-1226 (1996).

27. I. Schlupp, J. Parzefall, M. Schartl, Male mate choice in mixed bisexual/unisexual breeding complexes of Poecilia (Teleostei: Poeciliidae). Ethology 88, 215-222 (1991).

28. M.B. Ptacek, M.J. Childress, M.M. Kittell, Characterizing the mating behaviours of the Tamesí molly, Poecilia latipunctata, a sailfin with shortfin morphology. Anim. Behav. 70, 1339-1348 (2005).

29. C.A. Marler, M.J. Ryan, Origin and maintenance of a female mating preference. Evolution 51, 1244-1248 (1997). 
30. K. Witte, M.J. Ryan, Male body length influences mate-choice copying in the sailfin molly Poecilia latipinna. Behav. Ecol. 9, 534-539 (1998).

31. D. Bierbach, C. Kronmarck, C. Hennige-Schulz, S. Stadler, M. Plath, Sperm competition risk affects male mate choice copying. Behav. Ecol. Sociobiol. 65, 1699-1707 (2011).

32. D. Bierbach, A.M. Makowicz, I. Schlupp, H. Geupel, B. Streit, M. Plath, Casanovas are liars: behavioral syndromes, sperm competition risk, and the evolution of deceptive male mating behavior in live-bearing fishes. F1000Research 2, 75 (2013).

33. D.E. Broadbent, Information processing in the nervous system. Science 150, 457-462 (1965).

34. R. Dukas, Behavioural and ecological consequences of limited attention. Philos. Trans. R. Soc. Lond. B Biol. Sci. 357, 1539-1547 (2002).

35. K.U. Heubel, K. Hornhardt, T. Ollmann, J. Parzefall, M.J. Ryan, I. Schlupp, Geographic variation in female mate-copying in the species complex of a unisexual fish, Poecilia formosa. Behav. 145, 1041-1064 (2008).

36. M. Plath, S. Richter, R. Tiedemann, I. Schlupp, Male fish deceive competitors about mating preferences. Curr. Biol. 18, 1138-1141 (2008).

37. I. Schlupp, C. Marler, M.J. Ryan, Benefit to male Sailfin Mollies of mating with heterospecific females. Science 263, 373-374 (1994).

38. A. Mack, Z. Pappas, M. Silverman, R. Gay, What we see: inattention and the capture of attention by meaning. Conscious. Cogn. 11, 488-506 (2002).

39. M. Koivisto, A. Revonsuo, The effects of perceptual load on sematic processing under inattention. Psychon. Bull. Rev. 16, 864-868 (2009).

40. P. Maciej, A. Patzelt, I. Ndao, K. Hammerschmidt, J. Fischer, Social monitoring in a multilevel society: a playback study with male Guinea baboons. Behav. Ecol. Sociobiol. 67, 61-68 (2013).

41. H. Meyer, Investigations concerning the reproductive behavior of Mollienisia "formosa." J. Genet. 36, 329-366 (1938). 
415 Table 1: Results from the statistical models on stimulus females and audience directed attention in both the intrasexual competition and potential mate trials.

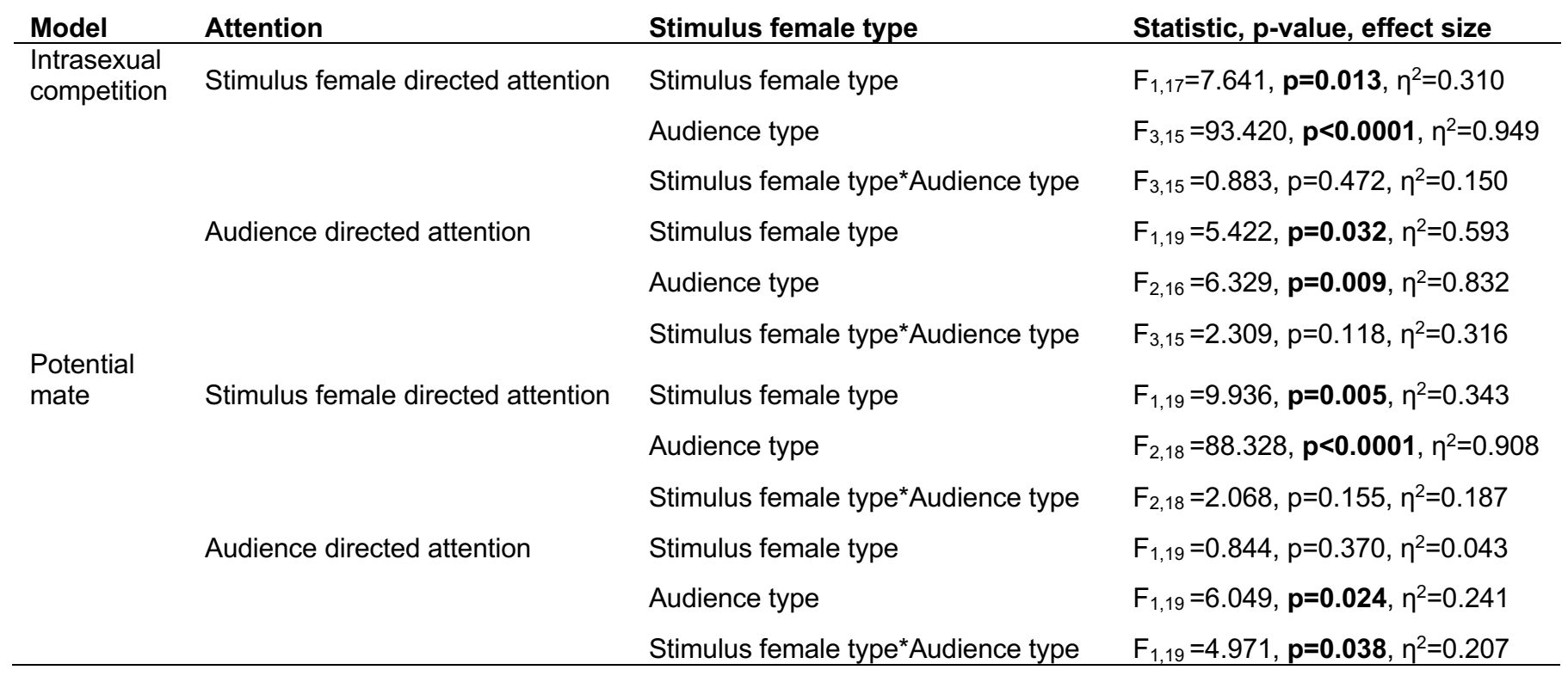


419 Table 2 - Descriptive statistics for intrasexual competition effects on male attention according to 420 stimulus female and male audience body size. $\mathrm{N}=18$ for all groups.

421

\begin{tabular}{|c|c|c|c|c|c|c|}
\hline Stimulus female & Audience male & Time (s) & Mean & Std Dev & Minimum & Maximum \\
\hline \multirow{8}{*}{ Amazon molly } & \multirow{2}{*}{ Control } & Stimulus & 475.07 & 115.80 & 132.44 & 600.00 \\
\hline & & Audience & -- & -- & -- & -- \\
\hline & \multirow[t]{2}{*}{ Smaller male } & Stimulus & 326.59 & 87.45 & 137.99 & 439.88 \\
\hline & & Audience & 118.38 & 74.30 & 20.04 & 273.76 \\
\hline & \multirow[t]{2}{*}{ Equal-sized male } & Stimulus & 310.77 & 122.89 & 87.52 & 535.12 \\
\hline & & Audience & 127.90 & 64.21 & 33.34 & 276.93 \\
\hline & \multirow[t]{2}{*}{ Larger male } & Stimulus & 242.00 & 90.37 & 82.20 & 404.08 \\
\hline & & Audience & 195.47 & 105.95 & 59.35 & 438.29 \\
\hline \multirow[t]{8}{*}{ Sailfin molly } & \multirow[t]{2}{*}{ Control } & Stimulus & 517.95 & 87.82 & 227.45 & 592.34 \\
\hline & & Audience & -- & -- & -- & -- \\
\hline & \multirow[t]{2}{*}{ Smaller male } & Stimulus & 376.18 & 98.82 & 158.40 & 502.82 \\
\hline & & Audience & 95.44 & 67.92 & 15.72 & 266.92 \\
\hline & \multirow[t]{2}{*}{ Equal-sized male } & Stimulus & 335.76 & 119.76 & 57.50 & 553.31 \\
\hline & & Audience & 119.29 & 68.40 & 21.35 & 248.85 \\
\hline & \multirow[t]{2}{*}{ Larger male } & Stimulus & 335.07 & 111.47 & 66.27 & 527.25 \\
\hline & & Audience & 137.68 & 77.17 & 25.98 & 327.79 \\
\hline
\end{tabular}


423 Table 3 - Descriptive statistics for female species effects on male attention according to stimulus and 424 audience female species. $\mathrm{N}=20$ for all groups.

425

\begin{tabular}{lllllll}
\hline Stimulus female & Audience female & Time (s) & Mean & Std Dev & Minimum & Maximum \\
\hline Amazon molly & Control & Stimulus & 504.64 & 71.31 & 339.20 & 600.00 \\
& & Audience & -- & -- & -- & -- \\
& \multirow{4}{*}{ Amazon molly } & Stimulus & 276.21 & 107.20 & 43.11 & 425.86 \\
& & Audience & 113.02 & 63.99 & 7.76 & 243.79 \\
& \multirow{4}{*}{ Sailfin molly } & Stimulus & 205.50 & 76.16 & 38.74 & 329.76 \\
& \multirow{3}{*}{ Sailfin molly } & Audience & 224.23 & 125.24 & 30.99 & 519.75 \\
& \multirow{3}{*}{ Amazon molly } & Stimulus & 531.58 & 102.47 & 252.97 & 660.46 \\
& & Audience & -- & -- & -- & - \\
& \multirow{2}{*}{ Sailfin molly } & Stimulus & 315.47 & 131.42 & 110.84 & 527.84 \\
& & Audience & 140.43 & 116.41 & 4.75 & 460.13 \\
& Stimulus & 308.02 & 128.83 & 118.59 & 527.84 \\
& Audience & 162.45 & 141.01 & 19.79 & 458.47 \\
\hline
\end{tabular}

426 
Fig. 1: The proportion of time focal males spent with each female when exposed to different male audiences (no audience = blue; smaller male = purple; male equal in size = green; larger male $=$ yellow). Dashed line indicates the maximum amount of time a male could spend with either or both females.

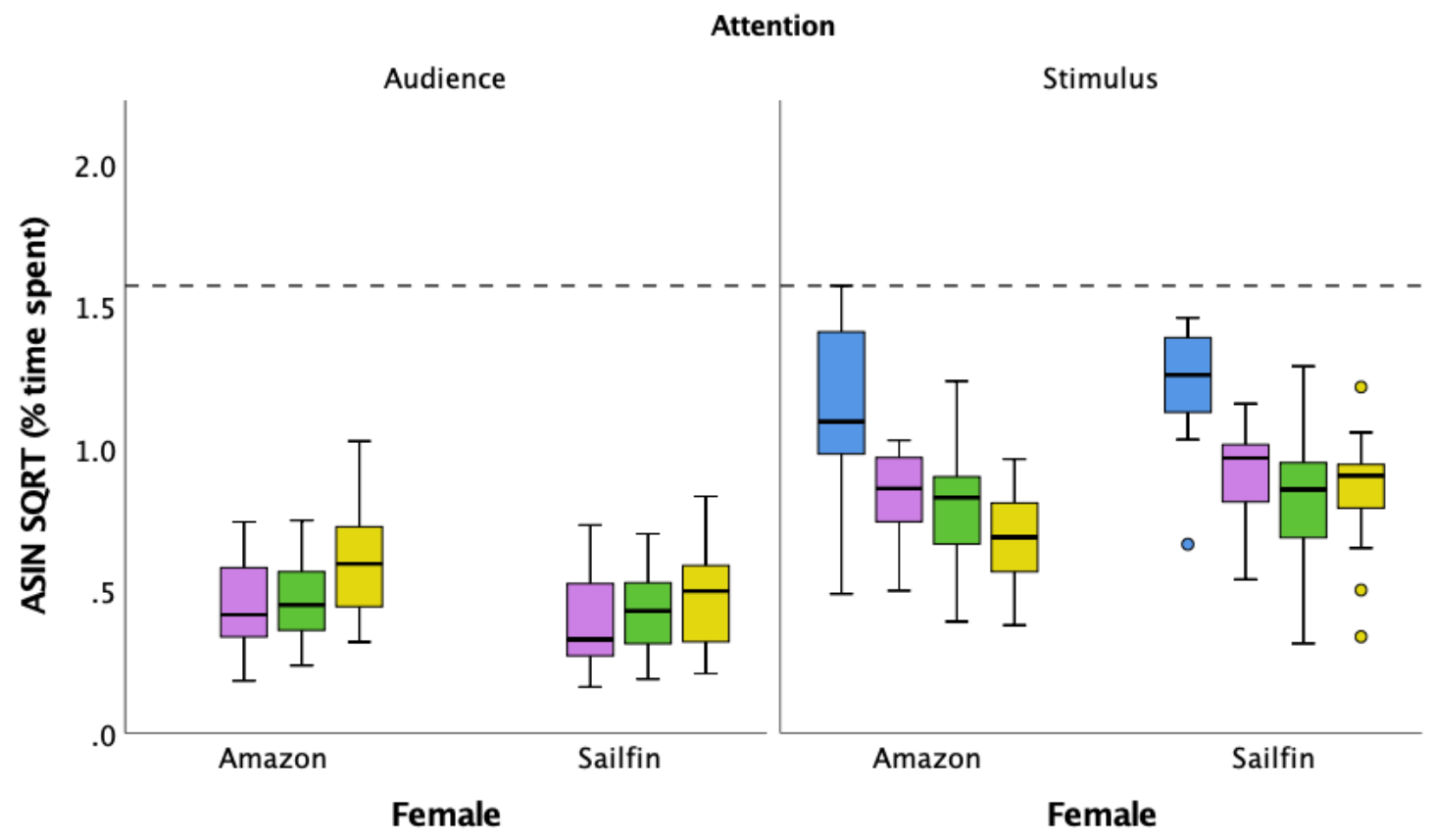


Fig. 2: The proportion of attention focal males spent with each female when exposed to different female audiences (no audience = blue; sailfin molly female $=$ pink; Amazon molly $=$ red). Dashed line indicates the maximum amount of time a male could spend with either or both females.

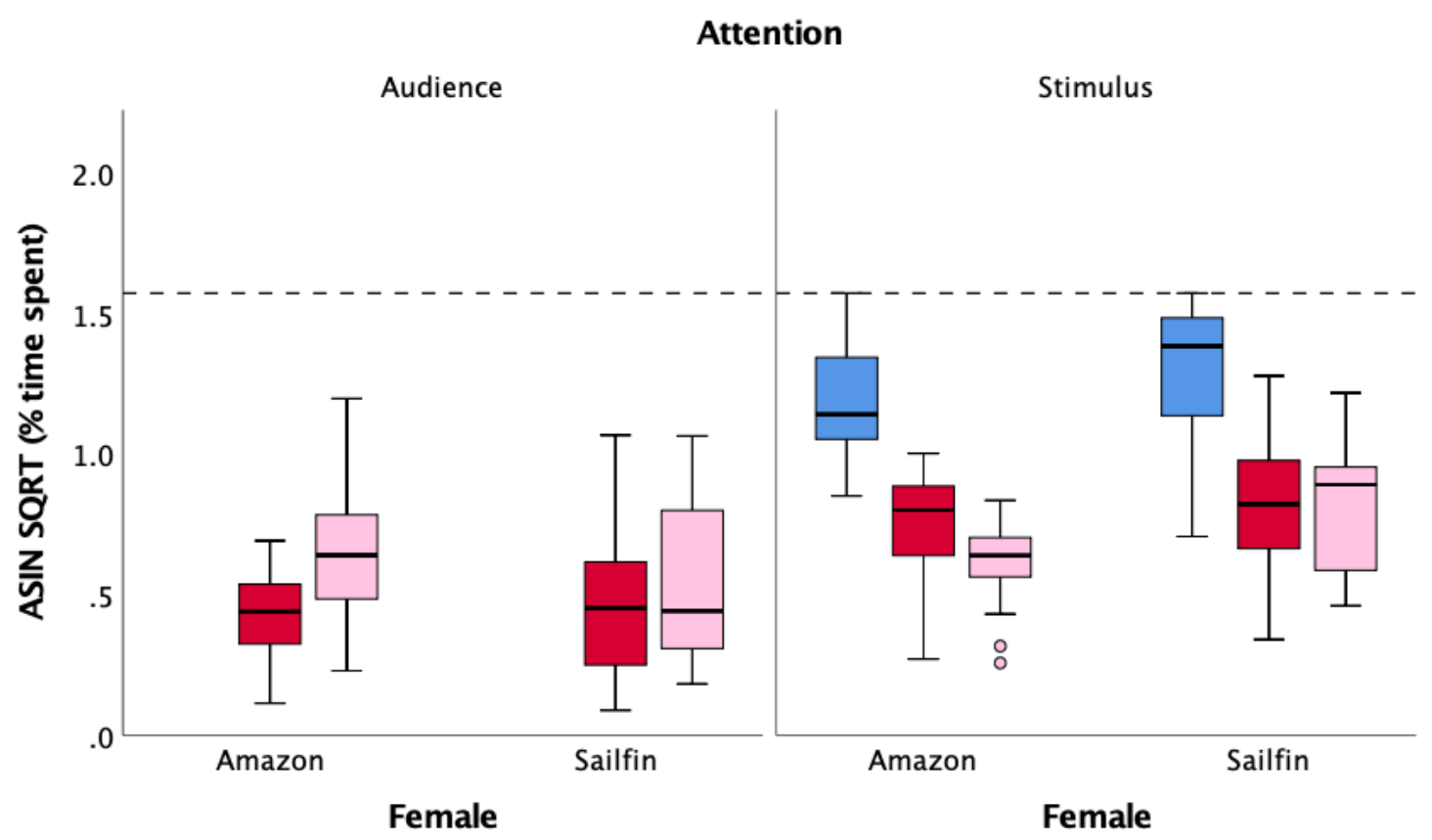

